\title{
Polarization field in a single-valley strongly-interacting 2D electron system
}

\author{
V. T. Dolgopolov and A. A. Shashkin \\ Institute of Solid State Physics, Chernogolovka, Moscow District 142432, Russia
}

\begin{abstract}
The magnetic field of complete spin polarization is calculated in a disorderless single-valley strongly-interacting 2D electron system. In the metallic region above the Wigner-Mott transition, non-equilibrium spin states are predicted, which should give rise to hysteresis in the magnetization.
\end{abstract}

PACS numbers: 73.50.Jt, 71.30.+h

A transition from the high conductivities $\sigma \gg e^{2} / h$ to the low conductivities $\sigma \ll e^{2} / h$ that is observed with decreasing electron density in disordered $2 \mathrm{D}$ electron systems was first interpreted as the Anderson metalinsulator transition [1]. The interpretation changed after the appearance of the weak localization theory [2] and scaling hypothesis [3]. According to the latter, there is no metallic state in disordered infinite $2 \mathrm{D}$ electron systems at zero temperature. As a result, the transition observed in experiment was referred to as "apparent" metal-insulator transition.

The next interpretation change was caused by the finding that the temperature dependence of the conductivity in low-disordered 2D electron systems changes sign at a critical density attributed to the metal-insulator transition point [4]. The observed critical density corresponds to strong electron-electron interactions, whereas noninteracting or weakly-interacting $2 \mathrm{D}$ systems are considered in the scaling concept. Moreover, the spin susceptibility and effective mass in the least-disordered 2D electron systems were found to increase at low densities with a tendency to diverge at some electron density that is weakly dependent on disorder [5, 6]. This indicates that the metal-insulator transition observed in a number of 2 D electron systems is driven by interactions [7], while the disorder is of minor importance. Additional confirmation for this statement is provided by the observation of the critical increase of the effective mass with increasing interactions in the $2 \mathrm{D}$ fermion system composed of $\mathrm{He}^{3}$ atoms [8].

There has been published a good deal of experimental and theoretical work on the metal-insulator transition in two dimensions and related phenomena (see, e.g., experimental reviews [9-12] and theoretical publications [13 17]). The key question is whether the metal-insulator transition observed in low-disordered 2D electron systems is a transition to the Wigner crystal. The mass divergence when the crystallization point is approached on the metallic side was already demonstrated in the paper [18] using Gutzwiller's theory. Recently, many properties of strongly-interacting 2D and 3D systems have been explained within the concept of the Wigner-Mott transition using dynamical mean-field theory [17].

In this paper, we study the magnetic field of full spin polarization $B_{c}$ as a function of electron density $n_{s}$ for the Wigner-Mott transition. The magnetic field is assumed to be parallel to the $2 \mathrm{D}$ electron system and act on electron spins only. We use a lattice model [18] by adapting the results of the papers [19, 20] to the problem to be solved. The real $2 \mathrm{D}$ electron system is replaced by lattice sites with density $n_{s}$. If for the lattice introduced there is an energy gap between the lowest and next bands, filling every site with one electron results in a Wigner crystal model. However, in the ground state, two electrons with anti-parallel spins can be located, despite repulsion, on each site with some probability depending on $n_{s}$. Such sites determine the portion of mobile electrons and, thus, the transport properties of the electron system. For the case of noninteracting electrons, the model used corresponds to the half-filled lowest energy band. The noninteracting electrons in zero magnetic field occupy all states up to the Fermi momentum $k_{F}$, each state being filled with spin-up and spin-down electrons. In the lattice model, the probability to find on each site two electrons is equal to $1 / 4$, which is the same as the probability to find on each site no electrons.

The momentum distribution function for the system with electron interactions in the metallic state is shown in Fig. 1(a) for zero magnetic field. All states are occupied up to the Brillouin zone boundary $k_{0}$. A jump of the distribution function with height $Z<1$ occurs at $k_{F}$. With decreasing electron density both $k_{0}$ and $k_{F}$ decrease, the ratio $k_{F} / k_{0}$ being constant. As the critical density $n_{c}$ is approached, the jump $Z \rightarrow 0$ and the distribution function $f^{u, d} \rightarrow 0.5$. The shaded region that spans up to $k_{0}$ in the figure corresponds to the Wigner crystal. The many-electron wave function [19] describes the superposition of the crystal state and the Fermi quasi-particles with an effective mass $m^{*}$ that is inversely proportional to the distribution function jump at the Fermi momentum $k_{F}: m^{*} / m=1 / Z$, where $m$ is the band mass.

The distribution function for electrons with different spins in magnetic fields is displayed in Fig. 1(b, c). Both the quasi-particle weight $Z$ and the Fermi momenta of quasi-particles with different spins are influenced by magnetic field. Evidently, the increase of magnetic field leads to $k_{F}^{u} \rightarrow k_{0}$ and $k_{F}^{d} \rightarrow 0$. In what follows we find that with increasing magnetic field the quasi-particle weight $Z$ increases (decreases) for $n_{s} \gg n_{c}\left(n_{s} \gtrsim n_{c}\right)$ until at some degree of spin polarization, the electron system reaches by jump full spin polarization in which case every lattice site is filled with one electron. The presence of the jump of the degree of spin polarization gives rise to hysteresis with decreasing magnetic field. 


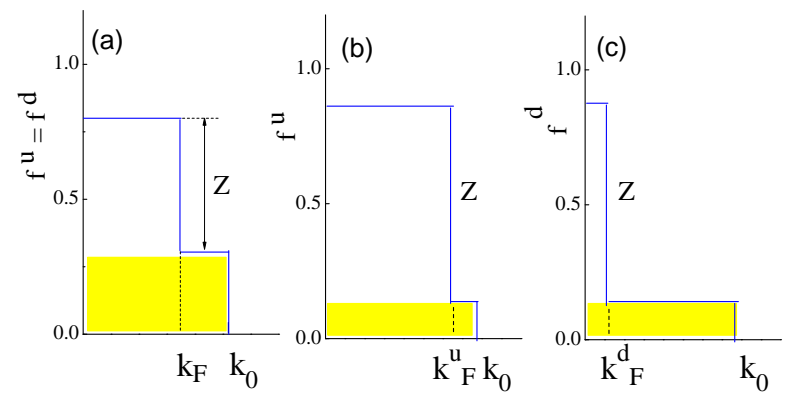

FIG. 1: Probability to occupy a state with momentum $k$ as a function of momentum in a certain direction: (a) for spinunpolarized system; (b) for spin-up electrons; (c) for spindown electrons. The shaded region corresponds to the Wigner crystal.

In accordance with the results 19, 21], the ground state-averaged Hamiltonian in a parallel magnetic field $B$ is written

$$
<H>=-\frac{Z}{4 D_{0}} n_{s}^{2}\left(1-p^{2}\right)+\frac{\nu \alpha e^{2}}{\kappa} n_{s}^{3 / 2}-\frac{p}{2} n_{s} g \mu_{B} B,
$$

where $\nu$ is the portion of doubly occupied sites $(0<\nu<$ $1 / 2), e$ is the electron charge, $\kappa$ is the dielectric constant, $D_{0}$ is the density of states of the spin-polarized noninteracting electrons. The degree of spin polarization $p=\left(n_{u}-n_{d}\right) / n_{s}$ is determined by the difference of the densities of spin-up and spin-down electrons, and the coefficient $\alpha$ is determined by the electron wave function on the lattice site. The kinetic energy is counted from the band-averaged energy for noninteracting electrons. The relation between $Z$ and $\nu$ taking account of correlations is as follows

$$
Z=\frac{2 \nu}{1-p^{2}}\left[(1+p-2 \nu)^{1 / 2}+(1-p-2 \nu)^{1 / 2}\right]^{2} .
$$

For $p=0$, Eqs. (11, 2) reduce to the known ones in zero magnetic field [18].

It is necessary to find a minimum of the expression (11) over $\nu$, regarding the relation (2):

$$
\frac{\partial<H\left(\nu, p, n_{s}\right)>}{\partial \nu}=0,
$$

which yields a dependence $\nu\left(p, n_{s}\right)$

$$
1-4 \nu+\frac{(1-2 \nu)(1-4 \nu)-p^{2}}{\left[(1-2 \nu)^{2}-p^{2}\right]^{1 / 2}}=2\left(\frac{n_{c}}{n_{s}}\right)^{1 / 2},
$$

where $n_{c}=\left(\alpha e^{2} D_{0} / 2 \kappa\right)^{2}$.

Note that for $p=0$, the ground state energy is a minimum if

$$
\nu=\frac{1}{4}\left[1-\left(\frac{n_{c}}{n_{s}}\right)^{1 / 2}\right] .
$$
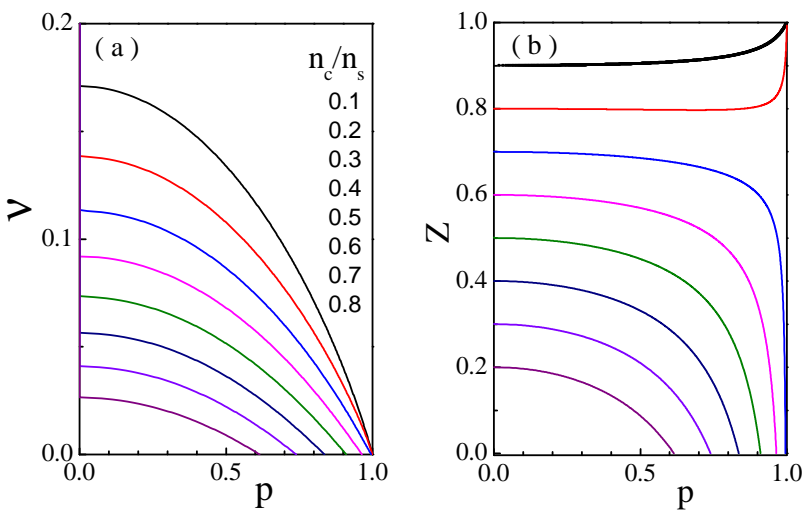

FIG. 2: (a) Portion of doubly occupied sites and (b) the quasiparticle weight versus spin polarization at different values of the interaction parameter.

At $n_{s} \rightarrow n_{c}$, both $\nu$ and $Z$ tend to zero, i.e., every lattice site is filled with one electron, and the quasi-particle mass diverges

$$
\frac{m^{*}}{m}=\frac{n_{s}}{n_{s}-n_{c}} .
$$

In the opposite limiting case of $n_{s} \rightarrow \infty$, the values $\nu$ and $Z$ are $\nu=1 / 4$ and $Z=1$, and the quasi-particle mass is equal to $m^{*}=m$.

The dependence $\nu(p)$ is shown in Fig. 2(a) for different values of the interaction parameter $n_{c} / n_{s}$. If $n_{s}>4 n_{c}$, the value $\nu$ zeroes only at $p=1$, whereas at $n_{s}<4 n_{c}$ the equality $\nu=0$ is the case over a whole range of $p$. We note that this fact is important for determining the dependence $B_{c}\left(n_{s}\right)$, and the density $n_{s}=4 n_{c}$ manifests itself as "apparent" critical density. The corresponding dependence $Z(p)$ is represented in Fig. 2(b). The value $Z$ increases (decreases) with spin polarization at $n_{s}>4 n_{c}$ $\left(n_{s}<4 n_{c}\right)$, attaining the limiting value $Z=1(Z=0)$. Interestingly, the spin states near $p=1$ turn out to be non-equilibrium states. If the dependence $\langle H(p)\rangle$ is two minima separated by a maximum, the electron system at $T=0$ gets to the deeper minimum in a magnetic field where the maximum disappears, which causes hysteresis.

We shall start by considering the high-density region $n_{s}>16 n_{c}$. In the spirit of the paper [22], one needs to find a minimum of the Hamiltonian (11, 2) over $p$ :

$$
\frac{\partial<H\left(\nu, p, n_{s}\right)>}{\partial p}=0,
$$

which yields

$$
\frac{2 \nu p}{\left[(1-2 \nu)^{2}-p^{2}\right]^{1 / 2}}=\frac{g \mu_{B} B D_{0}}{n_{s}} .
$$

By solving Eqs. (44 (8) with $p \rightarrow 1$, we get the polarization field

$$
B_{c}=\frac{n_{s}}{g \mu_{B} D_{0}}\left[1-2\left(\frac{n_{c}}{n_{s}}\right)^{1 / 2}\right] .
$$






FIG. 3: The ground state energy as a function of spin polarization at fixed $n_{c} / n_{s}=0.6$ for different magnetic fields. The minimum is marked by arrows.

The dependence obtained is the same as the one suggested earlier [5] and is similar to $B_{c}\left(n_{s}\right)$ observed in experiment. Obviously, Eq. (9) cannot describe the equilibrium value $B_{c}$ down to $n_{s}=4 n_{c}$, because the WignerMott transition in zero magnetic field occurs at the lower density $n_{s}=n_{c}$.

We solve the problem numerically at electron densities near $4 n_{c}$. There, the system behavior turns out to be hysteretic. Using the dependence $\nu(p)$ of Fig. 2, we calculate the dependence $\langle H(p)\rangle$ for a fixed value of the interaction parameter $n_{c} / n_{s}$ at different magnetic fields, as shown in Fig. [3 for $n_{c} / n_{s}=0.6$. In weak magnetic fields, the energy has a minimum corresponding to the realized degree of spin polarization. As the magnetic field is increased, the minimum turns into an inflection and disappears entirely. The inflection determines the upper polarization field at which the system state jumps to $p=1, \nu=0$. The degree of spin polarization first increases linearly with magnetic field, then turns up, and finally, the electron system reaches complete spin polarization by jump, as shown in Fig. [4 With decreasing magnetic field there arises hysteresis. The electron system stays in the $p=1$ state until the maximum at $p<1$ in the dependence $\langle H(p)\rangle$ (Fig. 31) disappears at the lower polarization field at which the system state jumps out of $p=1$ to close the hysteresis loop (Fig. 4). It is clear that the lower polarization field is given by Eq. (9) at $n_{s}>4 n_{c}$ and is equal to zero at $n_{s}<4 n_{c}$.

The dependence of the polarization field on electron density with increasing and decreasing magnetic field is displayed in Fig. 5 along with the effective mass as a function of electron density in zero magnetic field. Based on linear extrapolation of the high-density values, the field $B_{c}$ tends to zero at $n_{s} \approx 4 n_{c}$, which is well above the density where the effective mass in zero magnetic field diverges. Note that very low temperatures are needed to
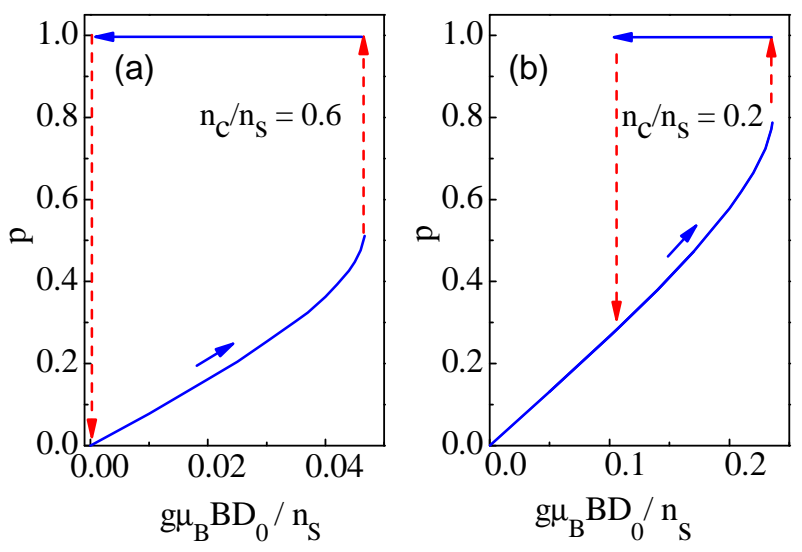

FIG. 4: Hysteresis of the degree of spin polarization with changing magnetic field at fixed (a) $n_{c} / n_{s}=0.6$ and (b) $n_{c} / n_{s}=0.2$.

observe the hysteresis in $B_{c}$ because the maximum in Fig. 3 is smeared due to thermal fluctuations already at $T \sim 0.1 \mathrm{~K}$.

In accordance with the solution, one might conclude that the magnetic field promotes crystallization at $n_{s}<$ $4 n_{c}$, whereas at higher densities, the system state in magnetic fields is the electron liquid. As a matter of fact, the complete filling of the first Brillouin zone in the model used means crystallization only if the energy gap is present for the model lattice. In turn, the energy gap is determined by electron-electron interactions on different lattice sites so that in the frames of the model [18 20], one cannot say whether or not the energy gap is present. Nevertheless, one can expect that the prediction is qualitatively correct: the gap in magnetic fields should survive at electron densities above $n_{c}$ and disappear at yet higher densities. Within the hysteresis un-

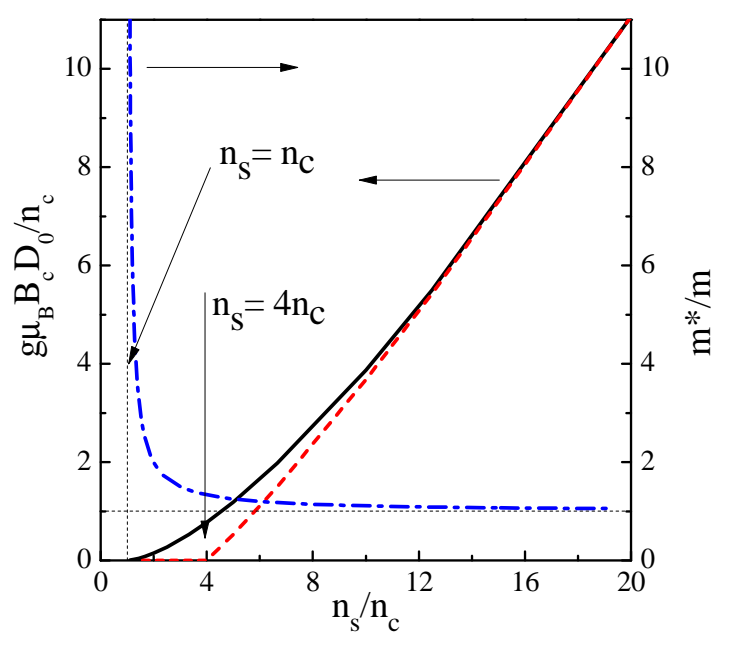

FIG. 5: Polarization field as a function of electron density with increasing (solid black line) and decreasing (dashed red line) magnetic field. Also shown by the dash-dotted blue line is the effective mass in $B=0$ versus electron density. 
certainty, the low-density part of $B_{c}\left(n_{s}\right)$ separates the correlated electron liquid and crystal and corresponds to the metal-insulator transition. The last fact is confirmed by comparison of the experimental results of Ref. [5] and Refs. [23, 24].

Another prediction of our calculations is that the effective mass in a single-valley electron system should decrease with magnetic field and reach the noninteracting electron mass at the onset of complete spin polarization if the system stays in the metallic state. The same behavior of the mass should be the case for full isospin polarization in a two-valley spin-polarized electron system. According to the statements of the papers [25, 26], the effect has been observed recently in the $2 \mathrm{D}$ electron system in AlAs quantum wells.

The magnetic field of full spin polarization as a function of electron density in the single-valley 2D electron system of GaAs/AlGaAs heterostructures was studied in Ref. [27]. Because of large thickness of the 2D system, the dependence $B_{c}\left(n_{s}\right)$ is strongly distorted by orbital effects. Nevertheless, its slope for low $B_{c}$ is in agreement with the slope expected from Eq. (9), and $B_{c}\left(n_{s}\right)$ extrapolates to zero at a finite density [10].

The most complete and detailed experimental information has been obtained for two-valley $2 \mathrm{D}$ electron systems. However, our calculations are not relevant for such systems, and comparison with those experiments is not justified.

It is interesting to compare our results with alterna- tive calculations. The behavior of the effective mass near the critical density of Eq. (6) has been reproduced in Ref. [17]. There, the dependence $B_{c}\left(n_{s}\right)$ has also been obtained which, unlike our results, does not reveal hysteresis.

It is worth noting that the polarization field $B_{c}$ versus $n_{s}$ for a two-valley $2 \mathrm{D}$ electron system has been calculated using quantum Monte-Carlo simulations [22]. In the clean limit $\left(k_{F} l \gg 1\right.$, where $l$ is the mean free path), the divergence of the mass and the critical $B_{c}\left(n_{s}\right)$ are not found. However, it is strange that the degree of spin polarization in the paper [22] is proportional to magnetic field, regardless of disorder and interaction strength.

In summary, we have calculated the dependence of the magnetic field of complete spin polarization on electron density for the Wigner-Mott transition in a single-valley $2 \mathrm{D}$ electron system. The following predictions of the model used have not yet been confirmed in experiment. In the metallic region above the Wigner-Mott transition, non-equilibrium spin states are expected, which should lead to a hysteretic behavior of the magnetization. Linear extrapolation to zero of the high-density values of the polarization field yields a critical density that exceeds the Wigner-Mott transition point by the factor of about four.

We gratefully acknowledge discussions with I. S. Burmistrov, V. Dobrosavljevic, and S. V. Kravchenko. This work was supported by RFBR, RAS, and the Russian Ministry of Sciences.
[1] T. Ando, A. B. Fowler, and F. Stern, Rev. Mod. Phys. 54, 435 (1982).

[2] L. P. Gor'kov, A. I. Larkin, and D. E. Khmel'nitskii, JETP Lett. 30, 228 (1979).

[3] E. Abrahams, P. W. Anderson, D. C. Licciardello, and T. V. Ramakrishnan, Phys. Rev. Lett. 42, 673 (1979).

[4] S. V. Kravchenko, G. V. Kravchenko, J. E. Furneaux, V. M. Pudalov, and M. D'Iorio, Phys. Rev. B 50, 8039 (1994).

[5] A. A. Shashkin, S. V. Kravchenko, V. T. Dolgopolov, and T. M. Klapwijk, Phys. Rev. Lett. 87, 086801 (2001).

[6] S. A. Vitkalov, H. Zheng, K. M. Mertes, M. P. Sarachik, and T. M. Klapwijk, Phys. Rev. Lett. 87, 086401 (2001).

[7] A. Punnoose and A. M. Finkelstein, Science 310, 289 (2005).

[8] A. Casey, H. Patel, J. Nyeki, B. P. Cowan, and J. Saunders, Phys. Rev. Lett. 90, 115301 (2003).

[9] S. V. Kravchenko and M. P. Sarachik, Rep. Prog. Phys. 67, 1 (2004).

[10] A. A. Shashkin, Physics-Uspekhi 48, 129 (2005).

[11] V. F. Gantmakher and V. T. Dolgopolov, PhysicsUspekhi 51, 3 (2008).

[12] A. A. Shashkin and S. V. Kravchenko, in "Understanding Quantum Phase Transitions", edited by L. D. Carr (Taylor \& Francis, Boca Raton, 2010).

[13] B. Spivak and S. A. Kivelson, Phys. Rev. B 70, 155114 (2004).

[14] S. Pankov and V. Dobrosavljevic, Phys. Rev. B 77,
085104 (2008).

[15] B. Spivak, S. V. Kravchenko, S. A. Kivelson, and X. P. A. Gao, Rev. Mod. Phys. 82, 1743 (2010).

[16] H. Terletska, J. Vucicevic, D. Tanaskovic, and V. Dobrosavljevic, Phys. Rev. Lett. 107, 026401 (2011).

[17] V. Dobrosavljevic, in "Conductor Insulator Quantum Phase Transitions", edited by V. Dobrosavljevic, N. Trivedi, and J. M. Valles Jr. (Oxford University Press, 2012).

[18] V. T. Dolgopolov, JETP Lett. 76, 377 (2002).

[19] M. C. Gutzwiller, Phys. Rev. A 137, 1726 (1965).

[20] W. F. Brinkman and T. M. Rice, Phys. Rev. B 2, 4302 (1970).

[21] See Eqs. (B4) and (B6) of Ref. 19].

[22] G. Fleury and X. Waintal, Phys. Rev. B 81, 165117 (2010).

[23] V. T. Dolgopolov, G. V. Kravchenko, A. A. Shashkin, and S. V. Kravchenko, JETP Lett. 55, 733 (1992).

[24] A. A. Shashkin, S. V. Kravchenko, V. T. Dolgopolov, and T. M. Klapwijk, Phys. Rev. Lett. 87, 266402 (2002).

[25] M. Padmanabhan, T. Gokmen, N. C. Bishop, and M. Shayegan, Phys. Rev. Lett. 101, 026402 (2008).

[26] T. Gokmen, M. Padmanabhan, and M. Shayegan, Phys. Rev. B 81, 235305 (2010).

[27] J. Zhu, H. L. Stormer, L. N. Pfeiffer, K. W. Baldwin, and K. W. West, Phys. Rev. Lett. 90, 056805 (2003). 